\title{
25G compared with 20G vitrectomy under Resight non-contact wide-angle lenses for Terson syndrome
}

\author{
XINBANG MAO and ZHIPENG YOU
}

Department of Ophthalmology, The Second Affiliated Hospital of Nanchang University, Nanchang, Jiangxi 330006, P.R. China

Received November 26, 2015; Accepted November 4, 2016

DOI: $10.3892 / \mathrm{etm} .2017 .4592$

\begin{abstract}
The aim of the present study was to compare the effectiveness of $25 \mathrm{G}$ vitrectomy to standard $20 \mathrm{G}$ vitrectomy for treatment of Terson syndrome under Resight non-contact wide-angle lenses. This was a case-control study of 20 patients with Terson syndrome (study group) that underwent $25 \mathrm{G}$ vitrectomy under Resight non-contact wide-angle lenses, with those of 20 matched patients that underwent $20 \mathrm{G}$ vitrectomy (control group). Medical records were reviewed from between July 2011 and October 2013. Data included results of the Early Treatment Diabetic Retinopathy Study examination, ophthalmology B-scan ultrasonography and fundus photography. The mean age, follow-up time, the preoperative visual acuity of LogMAR and the preoperative intraocular pressure (IOP) were all comparable in the two groups (all $\mathrm{P}>0.05$ ). There were statistically significant differences in postoperative visual acuity of LogMAR compared with preoperative visual acuity $(\mathrm{P}<0.001)$ in both groups, but no difference between the groups $(\mathrm{P}=0.845)$. However, the operative times $(13.5 \mathrm{~min}$ in study group vs. $42 \mathrm{~min}$ in control group) and post-operative IOP at day 1 (13.5 vs. $20 \mathrm{mmHg}$ ) were significantly reduced in the study group compared to the control group $(\mathrm{P}<0.001)$. Therefore, the present findings suggest that $25 \mathrm{G}$ Vitrectomy for Terson syndrome under Resight non-contact wide-angle lenses can achieve a significantly shorter operative time and lower post-operative IOP compared with 20G Vitrectomy.
\end{abstract}

\section{Introduction}

The syndrome of intra-vitreous bleeding in association with subarachnoid hemorrhage was first described by French ophthalmologist Albert Terson in 1900 (1). Terson syndrome now encompasses any intraocular hemorrhage associated with intracranial hemorrhage and elevated intracranial pressures (2-4), which often results in significant morbidity

Correspondence to: Dr Zhipeng You, Department of Ophthalmology, The Second Affiliated Hospital of Nanchang University, 1 Minde Road, Nanchang, Jiangxi 330006, P.R. China E-mail: mxb730828@126.com

Key words: Terson syndrome, non-contact wide angle lenses, 25G vitrectomy, 20G vitrectomy, intraocular pressure and subsequent decreased quality of life. Vitrectomy has been proposed to have a role in treating Terson syndrome, and numerous studies have reported good visual recovery following surgery (5-8). However, those studies were predominantly related to conventional $20 \mathrm{G}$ vitrectomy. Moreover, this technique has several complicating issues such as iatrogenic retinal breaks, entry site breaks and lengthy duration.

25G-transconjunctival sutureless vitrectomy (TSV) is a recently developed minimally invasive vitrectomy surgery system that has introduced a new approach to vitreoretinal surgery and shown advantages for some vitreoretinal surgeries (9-11). The advantages include decreased operative times in certain cases and decreased postoperative inflammation, early postoperative rehabilitation, improved patient comfort and minimal conjunctival damage (12). Comparison between $20 \mathrm{G}$ and $25 \mathrm{G}$ vitrectomy revealed that the time saved was localized to the 'initial' and 'final' steps of the procedure (13). Damage to the conjunctiva was also minimalized with a transconjunctival, sutureless approach (14). This may be of clinical significance in patients requiring glaucoma filtration surgery in the future. This benefit is also relevant to the limited number of patients undergoing multiple vitreoretinal procedures. Comparison of 25G-TSV wounds to conventional $20 \mathrm{G}$ wounds in the same patient revealed a much faster healing rate of 15 days as opposed to 6-8 weeks, using ultrasound biomicroscopy to assess the injury (15).

Recently, use of a wide-angle viewing system in vitrectomy surgery has become popular, as this option can easily provide a panoramic view of the surgical field (16). Two types of the wide-angle viewing system exist, with both contact and non-contact types available. The non-contact type is more popular because of the stability of the image against the tilt of the eyeball and the ease of manipulation (17). Resight non-contact wide-angled lenses are a new kind of operative viewing system. The simple operative procedure, wide observation, right stereo visual, non-contact wide-angle viewing system in cooperation with $25 \mathrm{G}$ vitrectomy may have the advantage of minimally invasive vitrectomy. The aim of this study was to compare the outcomes and therapeutic efficacy of $25 \mathrm{G}$ vitrectomy for Terson syndrome under Resight non-contact wide-angle lenses with the therapeutic efficacy of standard $20 \mathrm{G}$ vitrectomy.

\section{Materials and methods}

Materials. Between July 2011 and October 2013, we reviewed the records of 20 patients (28 eyes) diagnosed with Terson 
syndrome who had undergone $25 \mathrm{G}$ vitrectomy under Resight non-contact wide angle lenses (study group) and the records of 20 matched patients (27 eyes) that underwent standard $20 \mathrm{G}$ vitrectomy (control group). Patients in the control group were matched in terms of age, gender and cause of hemorrhage (Table I). This was a retrospective study that was approved by the ethics committee of Second Affiliated Hospital of Nanchang University [approval ID: 2011 (013); Nanchang, China]. Written consents were waived by the Ethics Committee.

Preoperative examinations. All patients underwent history consultation, an Early Treatment Diabetic Retinopathy Study examination was performed (18) and the best-corrected visual acuity (BCVA) (19) was recorded as LogMAR (20) visual examination. Intraocular pressure (IOP), ophthalmology B-scan ultrasonography and fundus photography were performed before the operation and IOP test was repeated at day 1 post operation. B-scan ultrasonography and fundus photography were performed to evaluate the posterior segment. Vitreous hemorrhage occurred in 24 and 24 eyes in study and control groups, respectively. The other 4 and 3 eyes were macular hemorrhage. The normal range for intraocular pressure was defined as between 10 and $21 \mathrm{mmHg}$.

Surgical methods for $25 G$ and $20 G$ vitrectomy. All surgeries were conducted by the same experienced doctor under local anesthesia (4 ml; $2 \%$ lidocaine) and using a CONSTELLATION 25G system (Alcon, Inc., Hünenberg, Switzerland) while Resight non-contact wide-angle lenses (Carl Zeiss Meditec AG, Jena, Germany) were used to observe fundus. The surgical method used was standardized three channels vitrectomy. Three channels were made with three $25 \mathrm{G}$ catheter needles on 4-mm site away from the limbus in the supertemporal, supernasal and infratemporal quadrant. The infusion pressure was maintained at $25 \mathrm{mmHg}$, the illumination level at $\mathrm{H} 4$, the suction pressure of $400 \mathrm{mmHg}$ and the cutting rate at 5,000 rpm. The vitrectomy consisted of core vitrectomy, the creation of a posterior vitreous detachment, peripheral vitrectomy and vitreous base shaving. The epiretinal membrane was peeled with $25 \mathrm{G}$ forceps. For eyes with epiretinal macular hemorrhage, the central vitreous body was cut first, then the epiretinal macular hemorrhage was removed by suction pressure. Exchange of intraocular fluid was performed in eyes with local retinal detachment. Finally, the three cannulas were removed. Eight eyes were tamponaded with Octafluoropropane C3F8 (Tianjin Jingming New Technology Development Co., Ltd., Tianjin, China); the other 20 eyes were not tamponaded with any material. Sclerotomies were sutured with 7-0 VICRYL (Ethicon US, LLC, Cincinnati, OH, USA), conjunctival peritomies were sutured with 8-0 VICRYL.

The 20G vitrectomy was performed using the standard 20G Accurus system (Alcon, Inc.). The surgery procedure was according to the routine operating methods (9). Nine eyes were tamponaded with $\mathrm{C} 3 \mathrm{~F} 8$ and 4 eyes tamponaded with silicone oil.

Statistical analysis. Statistical analysis was performed using SAS, version 9.2 (SAS Institute, Cary, NC, USA). Data are expressed as the mean \pm standard deviation or median (range), and Student's t-test was used to compare the difference between two groups or pre- vs. post-operation. The Chi-square test or Fisher's exact test was used to calculate the probability value for the comparison of dichotomous variables. A two-sided P-value of $<0.05$ was considered statistically significant.

\section{Results}

Patient characteristics and pre-operative data. The patient characteristics for the $25 \mathrm{G}$ vitrectomy (study) group and the $20 \mathrm{G}$ vitrectomy (control) group are shown in Table I. All patients recovered from a coma after neurosurgery or neurological treatment. The average interval between diagnosis of Terson syndrome and vitreous surgery was 6 weeks (range, 4-8 weeks). For patients that underwent the $25 \mathrm{G}$ vitrectomy, the preoperative visual acuity of LogMAR was 1.05 (range, 0.70-1.70), and for those who underwent the $20 \mathrm{G}$ vitrectomy it was 1.10 (range, $0.70-1.70)$. The intraocular pressure of all eyes was normal.

Operation time. The average operative time was $14 \mathrm{~min}$ (range, 10-16 min) for the 25G (study) group and it was $42 \mathrm{~min}$ (range, 30-55 min) for the $20 \mathrm{G}$ (control) group. The difference in the operative times between the two groups was statistically significantly different $(\mathrm{P}<0.001)$.

Postoperative visual acuity. Amongst the 25G (study) group, the preoperative visual acuity of $\log$ MAR was 1.05 (range, 0.70-1.70) while postoperative visual acuity of LogMAR was 0.26 (range, $0-0.60$ ); these pre- and post-operative values were significantly different $(\mathrm{P}<0.001)$. Amongst the 20G (control) group, the pre-operative visual acuity of LogMAR was 1.10 (range, 0.70-1.70), while postoperative visual acuity of LogMAR was 0.22 (range, 0.00-0.70); these pre- and post-op values were also significantly different $(\mathrm{P}<0.001)$. Significant visual improvement occurred in all eyes following vitrectomy.

Intraoperative fundus. In all eyes that underwent $25 \mathrm{G}$ vitrectomy, incomplete posterior vitreous detachment (PVD) was found in 18 eyes (64.3\%), epiretinal membrane in 8 eyes (28.6\%), macular epiretinal hemorrhage in 5 eyes $(17.8 \%)$. In all eyes that underwent $20 \mathrm{G}$ vitrectomy PVD was found in 17 eyes (63\%), epiretinal membrane in 9 eyes (33\%) and macular epiretinal hemorrhage in 5 eyes (19\%). There was not a significant difference between the two groups (Fig. 1).

Complications. Postoperativly, amongst the $25 \mathrm{G}$ vitrectomy group, cataracts occurred in 2 eyes (7.1\%), the intraocular pressure was normal at day 1 in all 28 eyes and retinal detachment and hemorrhage did not occur in any eyes. Amongst the patients that underwent the $20 \mathrm{G}$ vitrectomy (control group), cataracts occurred in 3 eyes $(11 \%)$ intraocular pressure was normal at day 1 in 16 eyes $(59.3 \%)$ and retinal detachment and hemorrhage in 3 eyes $(11.1 \%)$.

\section{Discussion}

In the present study, we compared the outcomes and therapeutic effects of 20 patients that underwent the $25 \mathrm{G}$ vitrectomy under Resight non-contact wide-angle lenses with a group of 20 matched patients that underwent the standard $20 \mathrm{G}$ vitrectomy operation (control group). A total of 28 eyes with 
Table I. Patient characteristics and outcomes by type of vitrectomy performed (25G vs. 20G vitrectomy) for Terson syndrome.

\begin{tabular}{|c|c|c|c|}
\hline Parameter & 25G study group & 20G control group & P-value \\
\hline Patients (n) & 20 & 20 & \\
\hline \multicolumn{4}{|l|}{ Gender } \\
\hline Male & 11 & 11 & - \\
\hline Female & 9 & 9 & - \\
\hline Age (years) & $40.0 \pm 6.6$ & $40.2 \pm 7.1$ & 0.963 \\
\hline \multicolumn{4}{|l|}{ Cause of hemorrhage } \\
\hline $\mathrm{CA}$ & 3 & 3 & - \\
\hline TA & 10 & 10 & \\
\hline $\mathrm{CH}$ & 2 & 2 & \\
\hline SSH & 5 & 5 & \\
\hline Follow-up (months) & $10.5(8-14)$ & $11(7-15)$ & 0.858 \\
\hline Disease eyes (n) & 28 & 27 & \\
\hline Left/Right & $13 / 15$ & $12 / 15$ & 0.883 \\
\hline Pre-operative BCVA & $1.05(0.70-1.70)$ & $1.10(0.70-1.70)$ & 0.986 \\
\hline Final BCVA & $0.26(0-0.60)$ & $0.22(0-0.70)$ & 0.845 \\
\hline Pre-operative IOP (mmHg) & $15(10-20)$ & $14.9(10.5-20.5)$ & $>0.05$ \\
\hline Operation times (min) & $13.5(10-16)$ & $42(30-55)$ & $<0.001$ \\
\hline Postoperative IOP at day 1 (mmHG) & $13.5(10-20)$ & $20(10-35)$ & $<0.001$ \\
\hline Normal IOP at day $1(10-21 \mathrm{mmHg})$ & $100 \%(28 / 28)$ & $59.3 \%(16 / 28)$ & $<0.001$ \\
\hline Intraoperative complication of retinal break & $0 \%(0 / 28)$ & $14.8 \%(4 / 28)$ & 0.111 \\
\hline
\end{tabular}

Continuous variables are provided as means with \pm standard deviation or median (range). CA, cerebral aneurysms; TA, traffic accidents; $\mathrm{CH}$, cerebral hemorrhage; SSH, spontaneous subarachnoid hemorrhage; IOP, intraocular pressure; BCVA, best-corrected visual acuity.
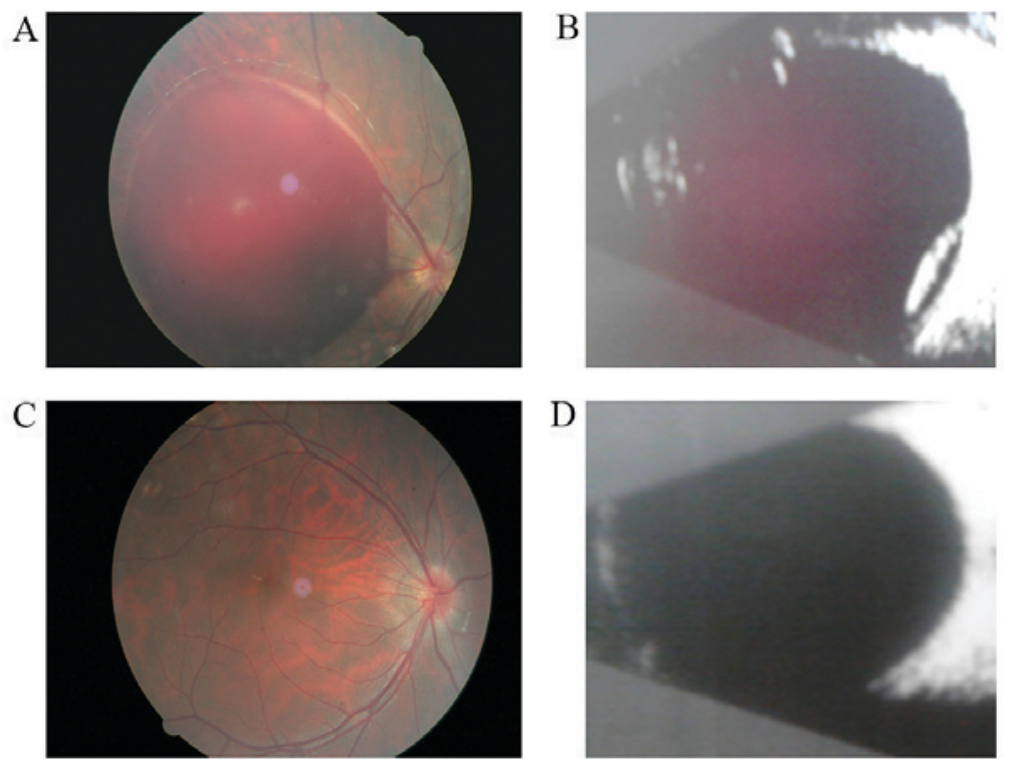

Figure 1. Fundus and ophthalmology B-scan ultrasonography findings from a woman who underwent 25G vitrectomy under Resight non-contact wide-angle lenses because of spontaneous subarachnoid hemorrhage. (A) Preoperative color fundus photograph shows epiretinal macular hemorrhage. (B) Ophthalmology B-scan ulrasonography shows arc light in the macular area. (C) Color fundus photograph obtained 8 months after surgery. The epiretinal macular hemorrhage had disappeared. (D) Ophthalmology B-scan ultrasonography obtained 8 months after surgery. The arc light has disappeared in the macular area. Best-corrected visual acuity improved from 0.7 before surgery to 0 at 8 months after surgery.

Terson syndrome underwent $25 \mathrm{G}$ vitrectomy and 27 underwent $20 \mathrm{G}$ vitrectomy using Resight non-contact wide-angle lenses. A significant therapeutic effect was achieved in both groups and the visual acuity was improved in all eyes after vitrectomy. The mean operative time was significantly shorter in the study group $14 \mathrm{~min}$ (range, 10-16 $\mathrm{min}$ ) compared with $42 \mathrm{~min}$ (range, 30-55 min) in the control group. The postoperative intraocular pressure was within the normal range at day 
1 in $100 \%$ of eyes in the study group and $59 \%$ in the control group. Slight inflammatory reaction occurred in all eyes in study group postoperatively, while several eyes in control group had inflammatory reaction a more severe, but this result comes from subjective feelings without objective indexes.

Vitrectomy has been shown to be effective at improving BCVA in previous studies in the majority of patients (7). For example visual acuity of 20/40 or better has been achieved in $76-93 \%$ of patients $(7,21,22)$. Variations in improving BCVA are apparently related to patient age and time elapsed between hemorrhage and surgery $(7,23)$. However, the majority of studies have used a $20 \mathrm{G}$ system. The results of the present study suggest that $25 \mathrm{G}$ vitrectomy produced similar improvements in visual acuity in patients with Terson syndrome to procedures involving 20G.

The main advantages suggested by these results of the $25 \mathrm{G}$ system over the standard $20 \mathrm{G}$ system are significantly shorter operation time and higher numbers of eyes with an intraocular pressure within the normal range one day after the procedure. A shorter operation time would be expected to decrease the cost of the procedure and is likely to be a major benefit to both the patient and the surgical team. However, there is some evidence that increased intraocular pressure after vitroretinal surgery might increase the risk of developing secondary glaucoma (24). Intraocular pressure often increases immediately after vitrectomy (25), and this was the case in this study. However, all of the eyes in the study group were within the normal range at day one but only $59 \%$ of those in the control group. Usually the increased intraocular pressure after vitrectomy is transient and easily managed (25). Long-term follow-up is needed to evaluate the risk to the eyes of this difference between the groups.

Despite no statistical difference in intraoperative complications between the groups, the result that there were no cases of retinal break in the study group but there were four in the control group suggests that a larger study is needed to fully evaluate the differences in complications between the groups. The higher number of retinal breaks suggests that the control group is likely to be at greater risk of retinal detachment, although the true risk this poses will have to be evaluated by long-term follow-up. The occurrence of retinal detachment with or without proliferative viteroretinopathy in Terson syndrome has been described previously (26). Retinal detachment is one of the more common postoperative complications reported in 9 and $32 \%$ of eyes in other studies where Terson syndrome was treated by vitrectomy $(7,22)$. However, none of the eyes in this study exhibited retinal detachment and vitreous hemorrhage after vitrectomy, which may be due to the small number of cases. Other common complications include cataracts, and in the present study cataracts occurred in two eyes tamponaded with $\mathrm{C} 3 \mathrm{~F} 8$ in the study group, a rate of $7 \%$. Visual acuity improves after phacoemulsification (7). The development of nuclear sclerosis following vitreous surgery is a well-documented complication (22). It has been noted that $7-10 \%$ of patients developed cataracts after vitrectomy for Terson syndrome, and other studies have reported rates of 16 , 20 and $32 \%(7,22,23)$.

In the present study, the epiretinal membrane was observed in 8 eyes $(28.6 \%)$ in the $25 \mathrm{G}$ virectomy group and in 9 eyes $(33 \%)$ in the $20 \mathrm{G}$ vitrectomy group. Other investigations have also observed the occurrence of the membrane in Terson syndrome $(7,21,22,27)$. It is likely that some gaps developed in the internal limiting membrane during the evolution of a dome-shaped hemorrhage, and later the proliferation of glial cells penetrate these gaps resulting in the epiretinal membrane. Another reason could be that the release of growth factors in the hemorrhage stimulates cell migration and proliferation.

The present study included some limitations. As a retrospective study the patients were not randomly allocated into groups rather they were assessed according to the treatment they received, this may have introduced some bias into the results. The study size was relatively small; a larger sample from multiple centers might provide statistical evidence of a difference in the rates of complications between the groups. The patients should be followed up for a longer period of time, this would provide more details about the true rate of complications that might occur many months after the procedure, and whether the visual improvements are long term.

This study suggests that, in comparison with $20 \mathrm{G}$ vitrectomy, 25G vitrectomy for Terson syndrome provides similar improvements in visual acuity but with shorter operation time and better intraocular pressure at day one. These results suggest that $25 \mathrm{G}$ vitrectomy should be considered for the treatment of Terson syndrome.

\section{Acknowledgements}

The authors thank the staff members of this trial, their colleagues and all the study staff for their efforts in collecting and ensuring the accuracy and completeness of all the data.

\section{References}

1. Kuhn F, Morris R, Witherspoon CD and Mester V: Terson syndrome. Results of vitrectomy and the significance of vitreous hemorrhage in patients with subarachnoid hemorrhage. Ophthalmology 105: 472-477, 1998.

2. Fountas KN, Kapsalaki EZ, Lee GP, Machinis TG, Grigorian AA, Robinson JS, Vergados I and Theodosiadis PG: Terson hemorrhage in patients suffering aneurysmal subarachnoid hemorrhage: Predisposing factors and prognostic significance. J Neurosurg 109: 439-444, 2008.

3. Stienen MN, Lücke S, Gautschi OP and Harders A: Terson haemorrhage in patients suffering aneurysmal subarachnoid haemorrhage: A prospective analysis of 60 consecutive patients. Clin Neurol Neurosurg 114: 535-538, 2012.

4. Rheinboldt M, Francis K, Parrish D, Harper D and Blase J: Terson syndrome in conjunction with ruptured intracranial aneurysm and penetrating intracranial injury: A review of two cases. Emerg Radiol 21: 215-218, 2014.

5. Kapoor S: Terson syndrome: An often overlooked complication of subarachnoid hemorrhage. World Neurosurg 81: e4, 2014.

6. Errera MH, Barale PO, Ounnoughene Y, Puech M and Sahel JA: 25-Gauge transconjunctival vitrectomy in a case of bilateral epiretinal membrane associated with a Terson syndrome. J Fr Ophtalmol 32: 268-272, 2009 (In French).

7. Garweg JG and Koerner F: Outcome indicators for vitrectomy in Terson syndrome. Acta Ophthalmol 87: 222-226, 2009.

8. Murjaneh S, Hale JE, Mishra S, Ling RH and Simcock PR: Terson's syndrome: Surgical outcome in relation to entry site pathology. Br J Ophthalmol 90: 512-513, 2006.

9. Gallemore R, Thomas E and Boyer DS: Minimally Invasive Vitreoretinal Surgery. Review of Ophthalmology 9: 11-16, 2002.

10. Fujii GY, De Juan E Jr, Humayun MS, Pieramici DJ, Chang TS, Awh C, Ng E, Barnes A, Wu SL and Sommerville DN: A new 25 -gauge instrument system for transconjunctival sutureless vitrectomy surgery. Ophthalmology 109: 1807-1813, 2002. 
11. Fujii GY, De Juan E Jr, Humayun MS, Chang TS, Pieramici DJ, Barnes A and Kent D: Initial experience using the transconjunctival sutureless vitrectomy system for vitreoretinal surgery. Ophthalmology 109: 1814-1820, 2002.

12. Chen E: 25-Gauge transconjunctival sutureless vitrectomy. Curr Opin Ophthalmol 18: 188-193, 2007.

13. Chang CJ, Chang YH, Chiang SY and Lin LT: Comparison of clear corneal phacoemulsification combined with 25-gauge transconjunctival sutureless vitrectomy and standard 20-gauge vitrectomy for patients with cataract and vitreoretinal diseases. J Cataract Refract Surg 31: 1198-1207, 2005.

14. Nagpal M, Wartikar S and Nagpal K: Comparison of clinical outcomes and wound dynamics of sclerotomy ports of 20,25, and 23 gauge vitrectomy. Retina 29: 225-231, 2009.

15. Zhengyu S, Fang W, Ying F and Qinghua Q: The experimental research of rabbit's sclerotomy sites undergoing transconjunctival sutureless vitrectomy. Curr Eye Res 32: 647-652, 2007.

16. Aras C, Ucar D, Koytak A and Yetik H: Scleral buckling with a non-contact wide-angle viewing system. Ophthalmologica 227: 107-110, 2012

17. Park SW, Kwon HJ, Kim HY, Byon IS, Lee JE and Oum BS: Comparison of scleral buckling and vitrectomy using wide angle viewing system for rhegmatogenous retinal detachment in patients older than 35 years. BMC Ophthalmol 15: 121, 2015.

18. Writing Committee for the Diabetic Retinopathy Clinical Research Network, Fong DS, Strauber SF, Aiello LP, Beck RW, Callanan DG, Danis RP, Davis MD, Feman SS, Ferris F, et al: Comparison of the modified early treatment diabetic retinopathy study and mild macular grid laser photocoagulation strategies for diabetic macular edema. Arch Ophthalmol 125: 469-480, 2007.
19. Pei-Pei W, Shi-Zhou H, Zhen T, Lin L, Ying L, Jiexiong O, Wen-Bo Z and Chen-Jin J: Randomised clinical trial evaluating best-corrected visual acuity and central macular thickness after 532-nm subthreshold laser grid photocoagulation treatment in diabetic macular oedema. Eye (Lond) 29: 313-322, 2015.

20. Mason JO III, Nixon PA and White MF: Intravitreal injection of bevacizumab (avastin) as adjunctive treatment of proliferative diabetic retinopathy. Am J Ophthalmol 142: 685-688, 2006.

21. Sharma T, Gopal L, Biswas J, Shanmugam MP, Bhende PS, Agrawal R, Shetty NS and Sanduja N: Results of vitrectomy in Terson syndrome. Ophthalmic Surg Lasers 33: 195-199, 2002.

22. Ritland JS, Syrdalen P, Eide N, Vatne HO and Øvergaard R: Outcome of vitrectomy in patients with Terson syndrome. Acta Ophthalmol Scand 80: 172-175, 2002.

23. Gnanaraj L, Tyagi AK, Cottrell DG, Fetherston TJ, Richardson J, Stannard KP and Inglesby DV: Referral delay and ocular surgical outcome in Terson syndrome. Retina 20: 374-377, 2000.

24. Tranos P, Asaria R, Aylward W, Sullivan P and Franks W: Long term outcome of secondary glaucoma following vitreoretinal surgery. Br J Ophthalmol 88: 341-343, 2004.

25. Costarides AP, Alabata P and Bergstrom C: Elevated intraocula pressure following vitreoretinal surgery. Ophthalmol Clin North Am 17: 507-512, v, 2004.

26. Velikay M, Datlinger P, Stolba U, Wedrich A, Binder S and Hausmann N: Retinal detachment with severe proliferative vitreoretinopathy in Terson syndrome. Ophthalmology 101: 35-37, 1994.

27. Yokoi M, Kase M, Hyodo T, Horimoto M, Kitagawa F and Nagata R: Epiretinal membrane formation in Terson syndrome. Jpn J Ophthalmol 41: 168-173, 1997. 